20

\title{
Терагерцовый микроскоп на основе эффекта твердотельной иммерсии для визуализации биологических тканей*
}

\author{
(C) Н.В Черномырдин ${ }^{1,2}$, А.С Кучерявенко ${ }^{1,2}$, Е.Н Римская ${ }^{2}$, И.Н Долганова ${ }^{2,3}$, \\ B.А Желнов ${ }^{2}$, П.А Каралкин ${ }^{4,5}$, А.А Грядунова ${ }^{4,6}$, И.В Решетов ${ }^{6}$, \\ Д.В Лаврухин ${ }^{7}$, Д.С Пономарев ${ }^{7}$, В.Е Карасик ${ }^{2}$, К.И Зайцев ${ }^{1,2}$ \\ ${ }^{1}$ Институт общей фризики им. А.М. Прохорова РАН, \\ 119991 Москва, Россия \\ ${ }^{2}$ Московский государственный технический университет им. Н.Э. Баумана, \\ 105005 Москва, Россия \\ ${ }^{3}$ Институт фозики твердого тела РАН, \\ 142432 Черноголовка, Россия \\ 4 Лаборатория биотехнологических исследований, 3D Bioprinting Solutions, \\ 115409 Москва, Россия \\ ${ }_{5}^{5}$ Национальный медицинский исследовательский центр радиологии Минздрава России, \\ 125284 Москва, Россия \\ ${ }^{6}$ Институт регенеративной медицины, Первый московский государственный медицинский университет \\ им. И.М. Сеченова Минздрава России, \\ 119146 Москва, Россия \\ ${ }^{7}$ Институт сверхвысокочастотной полупроводниковой электроники им. В.Г. Мокерова РАН, \\ 117105 Москва, Россия \\ e-mail: chernik-a@yandex.ru
}

Поступила в редакцию 18.11.2018 г.

В окончательной редакции 27.01.2019 г.

Принята к публикации 31.01.2019 г.

Предложен новый метод терагерцовой (ТГц) микроскопии для визуализации биологических тканей с субволновым пространственным разрешением. Он позволяет преодолеть дифракционный предел Аббе, обеспечивая субволновое разрешение за счет эффекта твердотельной иммерсии - сужения каустики электромагнитного пучка при его фокусировке в свободном пространстве на небольшом расстоянии (меньше длины волны) позади среды с высоким показателем преломления. Разработана экспериментальная установка, реализующая предложенный метод и использующая лампу обратной волны и ячейку Голея в качестве источника и детектора ТГц излучения. В данной установке для реализации эффекта твердотельной иммерсии излучение фокусируется позади кремниевой полусферы. Экспериментально продемонстрировано рекордно высокое пространственное разрешение для оптических систем на основе эффекта твердотельной иммерсии $-0.15 \lambda$ (измерения проводились на длине волны $\lambda=500 \mu \mathrm{m}$ для границы перехода металл-воздух в качестве тестового объекта). Микроскопия на основе эффекта твердотельной иммерсии не предполагает применение диафрагм или других видов ближнепольных зондов для достижения субволнового разрешения и соответственно исключает связанные с отмеченными элементами энергетические потери. Метод был применен для визуализации мягких тканей, что позволило продемонстрировать его перспективность для биологии и медицины.

DOI: $10.21883 /$ OS.2019.05.47665.14-19

\section{Введение}

С конца XX века наблюдается значительное развитие методов терагерцовой (ТГц) визуализации [1]. Среди приложений ТГц изображающих систем особый интерес представляет медицинская диагностика $[2,3]$ и, в частности, дифференциация здоровых тканей и злокачественных новообразований различной нозологии и локали-

\footnotetext{
* The 22nd Annual Conference Saratov Fall Meeting 2018 (SFM'18): VI International Symposium „Optics and Biophotonics“ and XXII International School for Junior Scientists and Students on Optics, Laser Physics \& Biophotonics, September 24-29, 2018, Saratov, Russia. https://www.sgu.ru/structure/fiz/saratov-fall-meeting/previousconferences/sara
}

зации с использованием ТГц имиджинга на основе эндогенных (естественных) маркеров [4-6]. Большинство современных систем для ТГц визуализации основаны на традиционных линзовых и зеркальных фокусирующих оптических элементах, для которых характерно дифракционно-ограниченное пространственное разрешение. Даже для ТГц оптической системы с максимально высокой числовой апертурой (NA $=1.0$ в случае фокусировки излучения в свободном пространстве) дифракционный предел пространственного разрешения Аббе составляет $\approx 0.61 \lambda$, где $\lambda-$ длина волны излучения в свободном пространстве [7]. Поскольку длина волны ТГц излучения составляет несколько сотен микрометров, 
явление дифракции накладывает значительные ограничения на пространственное разрешение ТГц визуализации в целом и точность определения границ злокачественных новообразований в частности. Ввиду отмеченного разработка новых методов ТГц визуализации с субволновым пространственным разрешением является важным направлением развития ТГц науки и техники.

К настоящему моменту предложено несколько методов высокоразрешающей ТГц визуализации. Среди них отметим ТГц цифровую голографию [8] и метод синтеза апертуры [9]. Эти подходы позволяют достичь субволнового разрешения, однако требуют сложных вычислений для решения обратных задач. В свою очередь методы ТГц ближнепольной сканирующей зондовой микроскопии позволяют преодолеть дифракционный предел и достичь впечатляющих значений пространственного разрешения до $10^{-2}-10^{-3} \lambda[10-12]$. Тем не менее они предполагают детектирование очень слабых сигналов, рассеянных субволновыми зондами, расположенными в плоскости объекта, и соответственно требуют применения мощных источников и чувствительных детекторов ТГц излучения, которые до сих пор остаются громоздкими и дорогостоящими.

Другая группа методов построения высокоразрешающих ТГц изображающих систем основана на эффекте локализации электромагнитного поля позади диэлектрических объектов различной геометрии [13]. В частности, для достижения субволнового разрешения могут быть использованы субволновые каустики ТГц излучения, формируемые позади мезомасштабных диэлектрических или металлических частиц (например, сфер, кубиков или асимметричных частиц) - так называемые „тераструи“ (tera-jets) [14-18]. Так, в работах [19,20] достигнуто пространственное разрешение до $0.5 \lambda$, при этом использовались мезоразмерные частицы, изготовленные как из однородного материала, так и из метаматериалов с искусственно подобранными диэлектрическими свойствами [21]. Другим перспективным методом улучшения пространственного разрешения является эффект твердотельной иммерсии, связанный с сужением каустики электромагнитного пучка при ее формировании в свободном пространстве на небольшом расстоянии (меньше длины волны) позади диэлектрической среды с высоким показателем преломления [22]. Особого внимания заслуживает метод микроскопии на основе эффекта твердотельной иммерсии. Впервые он был экспериментально реализован в видимом и инфракрасном (ИК) диапазонах $[23,24]$. В настоящее время эффект твердотельной иммерсии нашел свое применение в микроскопии [23], оптическом хранении информации [24], визуализации на основе комбинационного рассеяния света [25], тепловизионных изображающих системах [26] и исследовании интегральных микросхем [27]. Позднее были предложены изображающие системы, реализующие принцип твердотельной иммерсии в миллиметровом [28] и ТГц диапазонах [29-32].
Среди существующих методов ТГц визуализации микроскопия на основе эффекта твердотельной иммерсии представляется наиболее перспективным для решения задач ТГц биофотоники благодаря сочетанию субволнового пространственного разрешения и высокой энергетической эффективности, обусловленной отсутствием потерь энергии при рассеянии на ближнепольных сканирующих зондах - субволновых диафрагмах и кантилеверах [33,34]. В то же время остаются актуальными проблемы адаптации данного метода для визуализации тканей различного типа, а также дальнейшее повышение пространственного разрешения, чувствительности и быстродействия. Одной из основных проблем, препятствующих применению ТГц микроскопии на основе эффекта твердотельной иммерсии в биофотонике, является фиксация мягких биологических тканей в предметной плоскости объектива в процессе сканирования их поверхности пучком сфокусированного ТГц излучения. Существующие конфигурации ТГц объективов на основе эффекта твердотельной иммерсии предполагают прямой контакт между оптическим элементом с высоким показателем преломления и движущейся тканью, как сообщается в работе [29], что может привести к искажениям изображения из-за непреднамеренного механического сжатия тканей и эффекта окклюзии [35]. Подобные искажения могут оказаться существенными при использовании ТГц микроскопии в задачах медицинской диагностики, в которых различия, наблюдаемые между тканями в нормальном состоянии и при наличии патологии, зачастую составляют несколько процентов $[2-6,36]$.

Целью настоящей работы являлось создание метода ТГц микроскопии мягких биологических тканей с субволновым пространственным разрешением на основе эффекта твердотельной иммерсии. Для этого была разработана экспериментальная установка, реализующая предложенный метод. В качестве источника ТГц излучения в установке используется лампа обратной волны, а в качестве детектора - ячейка Голея. Для реализации эффекта твердотельной иммерсии излучение фокусируется позади полусферы, изготовленной из высокорезистивного кремния (high-resistivity float-zone silicon, HRFZ-Si). Экспериментально показано, что разработанная система обеспечивает рекордно высокое пространственное разрешение среди существующих изображающих систем на основе эффекта твердотельной иммерсии, а именно $0.15 \lambda$ для интерфейса металл-воздух (измерения проводились на длине волны $\lambda=500 \mu \mathrm{m})$. Новый метод ТГц микроскопии применен для визуализации мягких тканей различного типа, полученные результаты свидетельствуют о перспективности ТГц микроскопии на основе эффекта твердотельной иммерсии для биологии и медицины. 


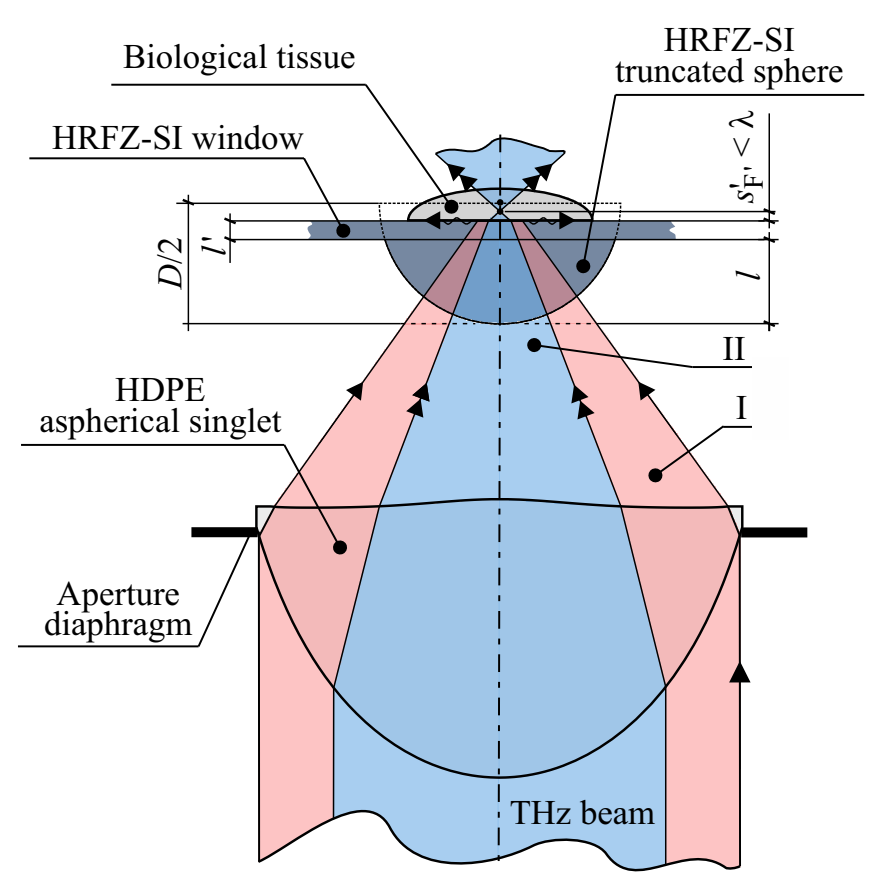

Рис. 1. Схема ТГц объектива, реализующего эффект твердотельной иммерсии. Он состоит из жестко закрепленных широкоапертурного полимерного асферического синглета и усеченной кремниевой сферы, а также подвижного кремниевого плоскопараллельного окна. В формировании каустики ТГц пучка участвуют как низкоапертурная составляющая пучка (I), проходящая через интерфейс „кремний-свободное пространстве“ (или „кремний-образец“), так и широкоапертурная составляющая (II), претерпевающая полное внутреннее отражение на данном интерфейсе.

\section{Экспериментальная установка}

На рис. 1 показана оптическая схема объектива, реализующего эффект твердотельной иммерсии, на которой отражен ход лучей. Каустика ТГц пучка в данной оптической системе формируется как частью пучка с низкой апертурой (I), проходящей через границу раздела „кремний-свободное пространство“, так и волнами полного внутреннего отражения (II), выходящими в свободное пространство при отражении широкапертурных компонент ТГц пучка от отмеченного интерфейса [37]. Так как значение критического угла полного внутреннего отражения зависит от оптических свойств исследуемого объекта, помещенного на поверхность объектива, то и вклады двух составляющих пучка - проходящей волны и волны полного внутреннего отражения - будут меняться. При этом и пространственное разрешение, и глубина резкости оптической системы зависят от оптических свойств исследуемого объекта $[33,38]$.

В состав данного объектива входят три оптических элемента:

- широкоапертурный синглет с двумя асферическими поверхностями, изготовленный из полиэтилена высокой плотности (HDPE);
- усеченная сфера, изготовленная из HRFZ-Si;

- плоскопараллельное окно, изготовленное из HRFZ-Si.

HRFZ-Si обладает высоким показателем преломления в ТГц диапазоне $n_{\mathrm{Si}}=3.415$. Асферический синглет имеет фокусное расстояние $15 \mathrm{~mm}$ и диаметр входного зрачка $25 \mathrm{~mm}$ [33], он позволит сформировать сходящийся пучок ТГц излучения дифракционного качества. Усеченная сфера имеет диаметр $D=10 \mathrm{~mm}$ и толщину $l=4.7 \mathrm{~mm}$. Ее сферическая поверхность расположена концентрично сходящемуся волновому фронту ТГц пучка, формируемому синглетом, в то время как ее плоская поверхность параллельна фокальной плоскости. Плоскопараллельное окно может перемещаться в латеральном направлении (поперек оптической оси объектива) и является одновременно частью единого оптического элемента, обеспечивающего субволновое пространственное разрешение, и держателем образца во время растрового сканирования его поверхности сфокусированным пучком ТГц излучения. Оно имеет толщину $l^{\prime}=0.25 \mathrm{~mm}$ и дополняет усеченную сферическую линзу практически до идеальной полусферы, толщина которой $l+l^{\prime}=4.95 \mathrm{~mm}$, а радиус $D / 2=5 \mathrm{~mm}$. Асферический синглет и усеченная сфера жестко закреплены, в то время как плоскопараллельное окно установлено на моторизованной двухкоординатной подвижке.

Такой подход к размещению биологических тканей предотвращает их сжатие (или другие виды механических деформаций), тем самым устраняя искажения ТГц изображений. Используя параксиальное приближение, можно оценить задний фокальный отрезок ТГц объектива, основанного на эффекте иммерсии:

$$
s_{F^{\prime}}^{\prime} \simeq\left(D / 2-l-l^{\prime}\right) n_{o b j} / n_{\mathrm{Si}}
$$

где $n_{o b j}-$ показатель преломления исследуемого объекта. Плотный контакт усеченной сферы и плоскопараллельного окна обеспечивает отсутствие потерь излучения, связанных с френелевским отражением. В настоящем исследовании не применялись никакие средства для уменьшения трения на интерфейсе между усеченной кремниевой сферой и плоскопараллельным окном, при этом не наблюдались повреждения оптических элементов, находящихся в контакте в ходе растрового сканирования или искажений ТГц изображений из-за образования воздушных зазоров между поверхностями, находящимися в контакте. Тем не менее систематическое исследование влияния перечисленных факторов на качество ТГц визуализации еще предстоит провести [31-33].

Схема ТГц микроскопа на основе эффекта твердотельной иммерсии представлена на рис. 2. В качестве источника непрерывного ТГц излучения с длиной волны $\lambda=500 \mu \mathrm{m}$ используется лампа обратной волны [39], в качестве детектора интенсивности ТГц излучения ячейка Голея (оптоакустический приемник) [40]. Поскольку ячейка Голея способна детектировать лишь 
THz SIL with motorized

$X-Y$ scanning system

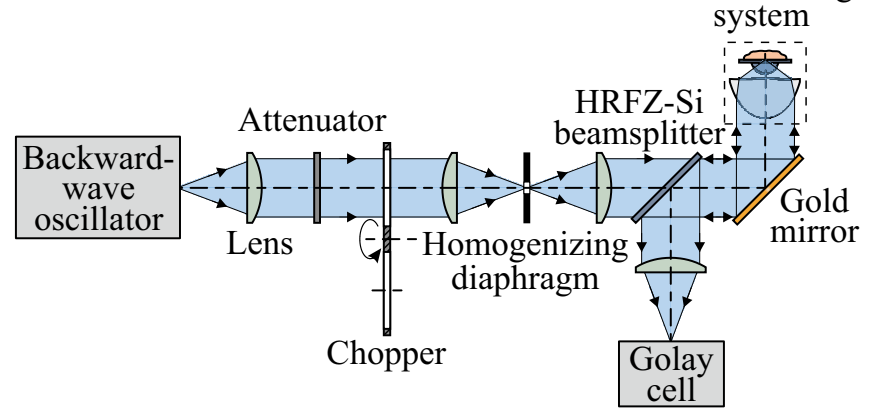

Pис. 2. Схема ТГц микроскопа на основе эффекта твердотельной иммерсии для визуализации биологических тканей с субволновым пространственным разрешением. Микроскоп работает по отраженному от поверхности образца излучению. В качестве источника непрерывного ТГц излучения используется лампа обратной волны, а в качестве детектора - ячейка Голея.

нестационарную интенсивность электромагнитной волны, для модуляции потока ТГц излучения используется механический чоппер с частотой $22 \mathrm{~Hz}$. Для предотвращения работы детектора в режиме насыщения пучок ТГц излучения пропускался через оптический ослабитель. Для гомогенизации интенсивности в сечении ТГц пучка в схеме микроскопа установлена телескопическая система, в фокальной плоскости которой расположена металлическая диафрагма диаметром $1 \mathrm{~mm}$, как показано на рис. 2.

Описанная конструкция имеет высокую чувствительность к угловой юстировке плоскопараллельного окна и кремниевой полусферы относительно оптической оси. Чтобы компенсировать остаточные погрешности юстировки, предложен подход к цифровому выравниванию яркости ТГц изображений по эталонной металлической поверхности. Для этого на верхнюю поверхность плоскопараллельного окна из HRFZ-Si нанесено титановое покрытие в форме квадратной рамки с внутренним размером (т.е. размером свободной от металла поверхности) $5 \times 5 \mathrm{~mm}$ и толщиной $300 \mathrm{~nm}$. Все ТГц изображения биологических тканей были получены с захватом металлической рамки по периметру. Коэффициент отражения титанового покрытия является однородным по всей своей площади, а соответственно информацию о неоднородности интенсивности изображения металлической рамки на ТГц микроскопических изображениях можно использовать для корректировки яркости. Опишем алгоритм корректировки яркости ТГц изображения.

- Измеряется интенсивность ТГц сигнала, отраженного от металлической рамки, вместе с интенсивностью исследуемого объекта.

- Далее для модельного описания коэффициентов спада яркости изображения по сравнению с ее реальными значениями используется линейный полином - уравнение двумерной плоскости. Его коэффициенты рассчитываются на основе яркости точек изображения металлической рамки, нормированной на максимальную яркость рамки:

$$
k(\mathbf{r})=I(\mathbf{r}) / I_{\max },
$$

где $\mathbf{r}$ - радиус-вектор в плоскости изображения.

- Далее на основе модели отклонения яркости изображения осуществляется ее корректировка - яркость каждого пикселя умножается на величину, обратнопропорциональную коэффициентам модели спада яркости изображения:

$$
I^{\prime}(\mathbf{r}) \equiv k^{-1} I(\mathbf{r})
$$

Быстродействие представленного метода регистрации ТГц изображений определяется несколькими факторами: чувствительностью и постоянной времени приемника ТГц излучения, размером изучаемой области и количеством регистрируемых точек, а также скоростью сканирования. Типичный размер сканируемой области объекта (с учетом захвата краев металлической рамки) составляет $6 \times 6 \mathrm{~mm}$. Шаг пространственного сканирования следует выбирать с учетом условия дискретизации Котельникова-Найквиста-Шеннона [41], принимая во внимание размер объекта исследования. Например, размеры жировых клеток лежат в диапазоне 5-75 $\mu \mathrm{m}$ для здоровых тканей и 100-200 $\mu$ m при патологии [42]. Если принять средний размер неоднородностей биологической ткани равный $100 \mu \mathrm{m}$, шаг пространственного сканирования по двум координатам стоит выбрать равным $50 \mu \mathrm{m}$. При данной конфигурации системы сканирования изображения размером $120 \times 120$ точек занимают порядка $40 \mathrm{~min}$. Благодаря широкой доступности в настоящее время быстродействующих АЦП и шаговых двигателей скорость сканирования ограничивается не временем перемещения плоскопараллельного окна с объектом относительно объектива, а временем регистрации одного пикселя ТГц изображения. Данный параметр определяется постоянной времени детектора излучения. Для ячейки Голея характерная чувствительность составляет $\sim 10^{-5} \mathrm{~V} / \mathrm{W}$, а постоянная времени $\sim 10^{-1} \mathrm{~s}$. Таким образом, сокращение продолжительности регистрации ТГц изображения можно достичь при использовании более быстродействующих детекторов, таких как охлаждаемый болометр (постоянная времени $\sim 10^{-6} \mathrm{~s}$ ) [43] или пироэлектрический приемник (постоянная времени $\sim 10^{-5} \mathrm{~s}$ ) [44]. В таком случае время регистрации одного кадра может достигать 5 min, будучи ограниченным скоростью перемещения шаговых двигателей.

Несмотря на небольшое быстродействие, ячейка Голея обладает достаточно высокой чувствительностью и в данной конфигурации обеспечивает отношение сигнал/шум $S N R$ порядка $10^{2}$. Дальнейшее увеличение $S N R$ возможно за счет увеличения времени накопления сигнала в каждой точке исследования, что, однако, существенно увеличит время регистрации ТГц изображения. 


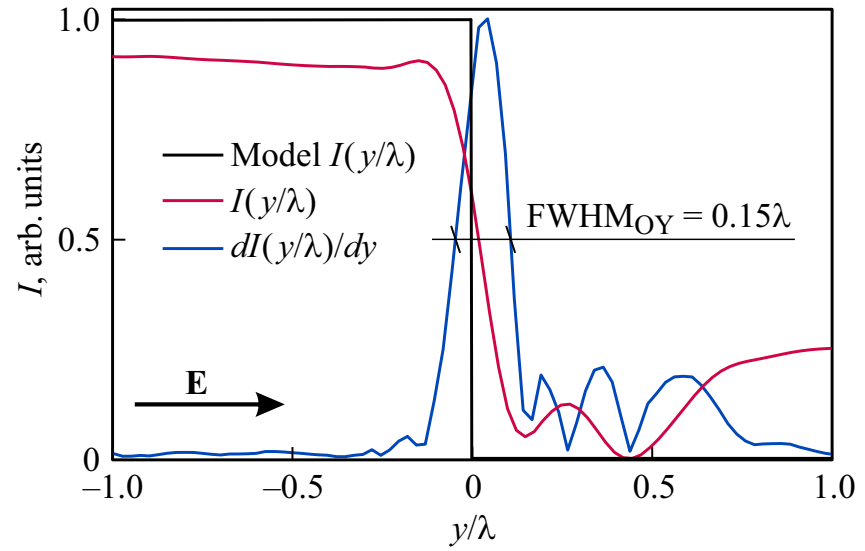

Pис. 3. Экспериментальная оценка пространственного разрешения ТГц микроскопа на основе эффекта твердотельной иммерсии для ТЕ-поляризации и длины волны излучения $\lambda=500 \mu \mathrm{m}$. На рисунке показан модельный профиль коэффициента отражения тестовой металлической полуплоскости $R(y / \lambda)$, профиль интенсивности ТГц изображения металлической полуплоскости $-I(y / \lambda)$, а также модуль его первой производной $-d I(y / \lambda) / d y$. Полуширина пика $d I(y / \lambda) / d y$ используется в качестве оценки разрешения.

Другим возможным способом может стать замена детектора на более чувствительный, например, охлаждаемый болометр (чувствительность $\sim 10^{2} \mathrm{~V} / \mathrm{W}$ ) [43].

\section{Экспериментальная оценка пространственного разрешения ТГц микроскопа}

Оценка пространственного разрешения ТГц микроскопа на основе эффекта твердотельной иммерсии проведена путем визуализации тестового объекта со ступенчатым изменением коэффициента отражения металлической полуплоскости, установленной позади подвижного плоскопараллельного окна. На рис. 3 показаны модельное сечение коэффициента отражения $R(y / \lambda)$ (оно имеет вид функции Хевисайда) и сечение интенсивности ТГц изображения тестовой металлической полуплоскости $I(y / \lambda)$. Пространственное разрешение изображающей системы оценивалось по полуширине (full-width at half-maximum - FWHM) пика на функции модуля первой производной профиля интенсивности $|d I(y / \lambda) / d y|$. Из рис. 3 видно, что разработанный ТГц микроскоп обеспечивает субволновое пространственное разрешение $0.15 \lambda$ для случая электромагнитной волны, поляризованной параллельно металлическому ножу. Также проведены исследования пространственного разрешения для ортогональной поляризации электромагнитного пучка (поляризация поперек металлического ножа), показавшие схожее пространственное разрешение $0.14 \lambda$ [33].
Достигнутое пространственное разрешение ТГц микроскопии значительно превосходит аналогичный показатель других систем на основе эффекта твердотельной иммерсии, которые были ранее предложены для работы в миллиметровом, ГГц и ТГц диапазонах, а именно -

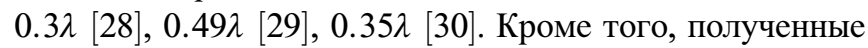
результаты сравнимы или превосходят значения пространственного разрешения изображающих систем на основе эффекта твердотельной иммерсии, ранее реализованных в видимом и ИК диапазонах, а именно $0.28 \lambda[26], 0.23 \lambda[45], 0.2 \lambda$ [46], 0.15-0.31 $\lambda$ [27]. На наш взгляд, столь высокое пространственное разрешение получено по двум причинам:

- использование асферического синглета позволило сформировать широкоапертурный пучок дифракционного качества, который обеспечивает высокое разрешение и без применения ближнефокусной кремниевой линзы [33];

- кремниевая полусфера позволила еще более повысить пространственное разрешение за счет эффекта твердотельной иммерсии $[30,33]$.

Дальнейшее улучшение пространственного разрешения возможно за счет использования синглета с большей числовой апертурой NA или выбора материала усеченной сферы с показателем преломления большим, чем y HRFZ-Si. Эффективный показатель преломления усеченной сферы может быть увеличен путем применения принципов замедления света в метаматериалах [47] или фотонно-кристаллических средах $[48,49]$.

Глубина резкости оптической системы, реализующей эффект твердотельной иммерсии, физически ограничена толщиной слоя свободного пространства позади плоскопараллельного окна из HRFZ-Si, в котором существует затухающее поле полного внутреннего отражения. На основе результатов теоретических исследований, проведенных ранее в работах $[30,38]$, можно заключить, что глубина резкости для рассмотренной оптической системы составляет около половины длины волны электромагнитного излучения в свободном пространстве. В случае, когда объектом исследования является биологическая ткань, глубина резкости существенно ниже из-за высокой концентрации воды.

Как было отмечено выше, поперечные и продольные размеры каустики ТГц пучка, формируемого позади кремниевой полусферы, зависят от условий полного внутреннего отражения на границе раздела „HRFZ-Siобъект“", а следовательно, и от оптических свойств самого объекта $[33,38]$. Подобная зависимость геометрии каустики от объекта является интересным явлением, присущим всем типам систем, основанных на эффекте твердотельной иммерсии. Дальнейшие исследования данного явления представляются крайне интересным направлением, однако оно выходит за рамки настоящей работы. 


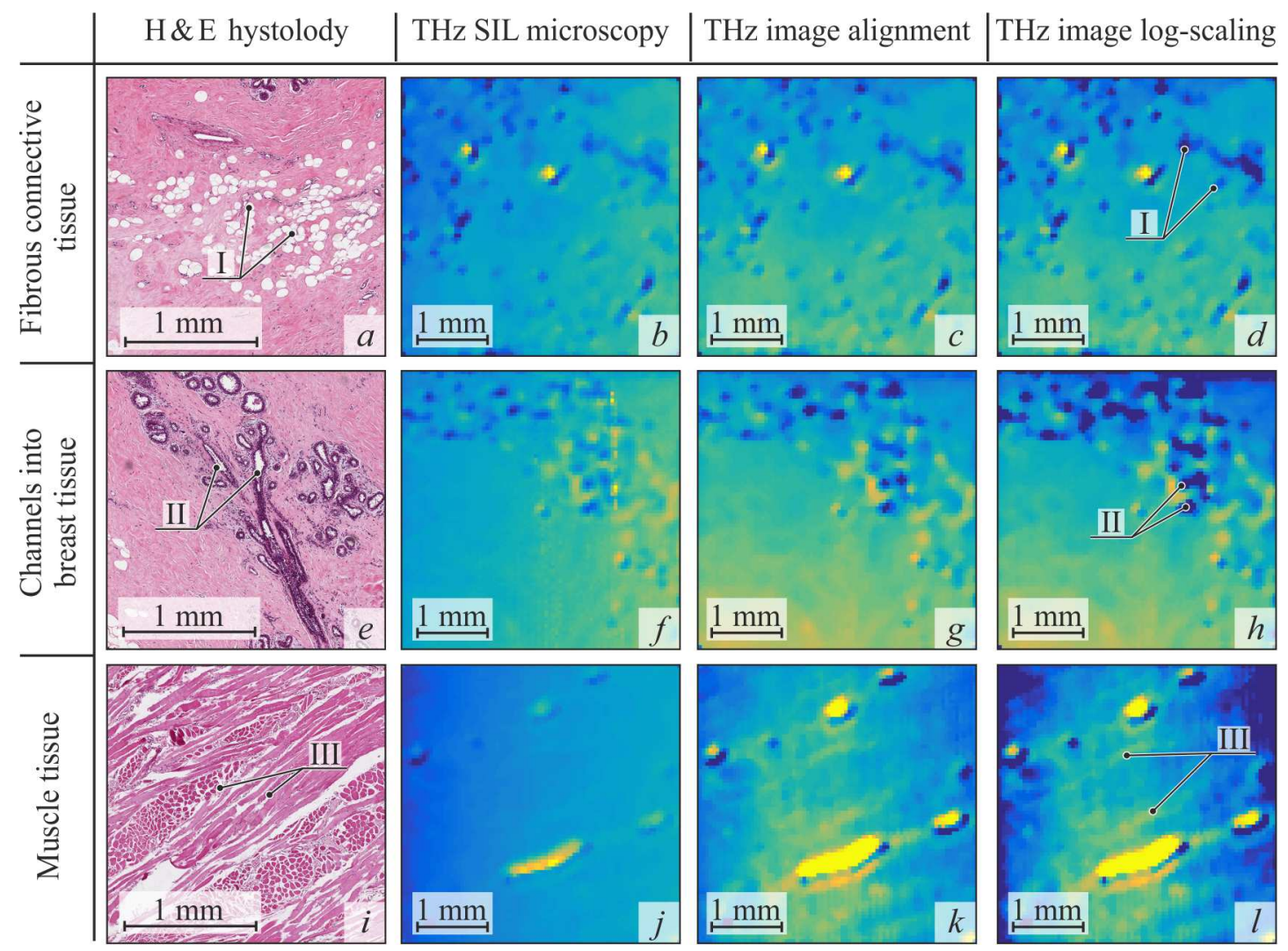

Рис. 4. Результаты ТГц микроскопии мягких биологических тканей на $\lambda=500 \mu \mathrm{m}: a, e, i-$ результаты гистологии тканей с окрасом гематоксилином и эозином (H\&E); $b, f, j-$ результаты ТГц микроскопии; $c, g, k$ - ТГц изображения после выравнивания яркости; $d, h, l-$ ТГц изображения в логарифмическом масштабе яркости. На $a, b, c, d$ показана строма молочной железы, где видны отдельные жировые клетки и их агломераты (I) в матрице соединительных тканей; на $e, f$, $g$, $h$ - расширенный проток молочной железы (II); на $i, j, k, l$ - поперечно-полосатые мышечные ткани языка с продольными и поперечными мышечными волокнами (III).

\section{Применение ТГц микроскопа для визуализации биологических тканей}

Чтобы экспериментально продемонстрировать возможность применения ТГц микроскопа, на основе эффекта твердотельной иммерсии, для задач медицинской диагностики, разработанная система применена для визуализации мягких биологических тканей человека ex vivo.

На рис. $4, b, f, j$ показаны ТГц изображения различных типов тканей человека. Для полученных изображений был применен метод компенсации остаточных аберраций, описанный в настоящей работе, результаты которого представлены на рис. $4, c, g, k$. Более того, изображения построены в логарифмическом масштабе, чтобы подчеркнуть низкоинтенсивные неоднородности (рис. $4, d, h, l)$. Все фрагменты тканей были иссечены в соответствии с первоначальным медицинским диагнозом. Толщина исследуемых образцов тканей составляла 3-5 mm. После ТГц измерений диагноз был уточнен путем гистологических исследований. На рис. 4, a,e, приведены результаты оптической микроскопии тканей (толщина срезов $4 \mu \mathrm{m}$ ) при фиксации гематоксилином и эозином (H\&E). Для предотвращения гидратации/дегидратации тканей при транспортировке и во время измерений образцы были покрыты желатиновой пленкой. Это позволило сохранить ТГц отклик тканей ex vivo неизменным по сравнению со свежеиссеченными тканями [50,51]. Приведенные ТГц изображения показывают, что разработанная система позволяет визуализировать следующие субволновые неоднородности тканей:

- включения зрелых жировых клеток и их агломератов (от 2 до 25 клеток) в строме молочной железы, образованной соединительными тканями (I на рис. 4, $a$ );

- расширенный проток молочной железы (II на рис. $4, e)$;

- продольные и поперечные мышечные волокна (III на рис. $4, i)$.

Рассмотренные структурные компоненты тканей имеют существенно субволновой размер, что не позволяет их визуализировать с применением классической линзовой или зеркальной ТГц оптики. Наблюдаемый контраст связан с различным содержанием воды в тканях и 
соответственно различными значениями коэффициента поглощения ТГц излучения тканями [3].

\section{Заключение}

В работе описан метод визуализации мягких тканей в ТГц диапазоне частот, сочетающий в себе принципы широкоапертурной асферической оптики и твердотельной иммерсии и позволяющий достичь пространственного разрешения $0.15 \lambda$ (для интерфейса металл-воздух). Получены ТГц изображения мягких тканей человека различной локализации ex vivo. Полученные результаты демонстрируют пространственно-неоднородный характер мягких биологических тканей на масштабах, определяемых ТГц длинами волн. Данный факт может накладывать ограничения на традиционное теоретическое представление взаимодействия ТГц излучения с тканями, основанное на допущении о пространственной однородности тканей и введении эффективного диэлектрического отклика тканей на основе релаксационных моделей комплексной диэлектрической проницаемости [3,52]. Следовательно, в таких системах значительное влияние на ТГц отклик биологических тканей должны оказывать эффекты рассеяния Ми, при этом физико-математические модели взаимодействия ТГц излучения с подобными тканями должны строиться на основе теории переноса излучения $[42,53,54]$. Результаты работы свидетельствуют о перспективности применения нового метода ТГц микроскопии для решения широкого спектра задач биологии и медицины.

\section{Финансирование работы}

Разработка метода ТГц микроскопии на основе эффекта твердотельной иммерсии и экспериментальные исследования, выполненные К.И. Зайцевым и Н.В. Черномырдиным, поддержаны Российским фондом фундаментальных исследований (РФФИ), проект № 18-2902060. Работа А.С. Кучерявенко, И.Н. Долгановой и В.Е. Карасика над обработкой и анализом экспериментальных данных поддержана РНФ, проект № 18-1200328.

\section{Соблюдение этических стандартов}

Все процедуры, выполненные в исследовании с участием людей, соответствуют этическим стандартам Хельсинкской декларации 1964 г. и ее последующим изменениям или сопоставимым нормам этики. От каждого из включенных в исследование участников было получено информированное добровольное согласие.

\section{Конфликт интересов}

Авторы заявляют, что у них нет конфликта интересов.

\section{Список литературы}

[1] Hu B.B., Nuss M.C. // Opt. Lett. 1995. V. 20. N 16. P. 1716 1718. doi 10.1364/OL.20.001716

[2] Yang X., Zhao X., Yang K., Liu Y., Liu Y., Fu W., Luo Y. // Trends Biotechnol. 2016. V. 34. N 10. P. 810-824. doi 10.1016/j.tibtech.2016.04.008

[3] Smolyanskaya O.A., Chernomyrdin N.V., Konovko A.A., Zaytsev K.I., Ozheredov I.A., Cherkasova O.P., Nazarov M.M., Guillet J.-P., Kozlov S.A., Kistenev Y.V., Coutaz J.-L., Mounaix P., Vaks V.L., Son J.-H., Cheon H., Wallace V.P., Feldman Y., Popov I. et al. // Prog. Quantum Electron. 2018. V. 62. P. 1-77. doi 10.1016/j.pquantelec.2018.10.001

[4] Woodward R.M., Wallace V.P., Pye R.J., Cole B.E., Arnone D.D., Linfield E.H., Pepper M. // J. Invest. Dermatol. 2003. V. 120. N 1. P. $72-78$. doi 10.1046/j.1523-1747.2003.12013.x

[5] Joseph C.S., Patel R., Neel V.A., Giles R.H., Yaroslavsky A.N. // J. Biophotonics. 2014. V. 7. N 5. P. 295-303. doi 10.1002/jbio.201200111

[6] Зайщев К.И., Черномырдин Н.В., Кудрин К.Г., Решетов И.В., Юрченко С.О. // Опт. и Спектр. 2015. T. 119. № 3. С. 404-410; Zaitsev K.I., Chernomyrdin N.V., Kudrin K.G., Reshetov I.V., Yurchenko S.O. // Opt. Spectrosc. 2015. V. 119. N 3. P. 404-410. doi 10.1134/S0030400X1509026X

[7] Novotny L., Hecht B. Principles of Nano-Optics. Cambridge: Cambridge University Press, 2012. doi 10.1017/CBO9780511794193

[8] Zhang Y., Zhou W., Wang X., Cui Y., Sun W. // Strain. 2008. V. 44. N 5. P. $380-385$. doi 10.1111/j.1475-1305.2008.00433.x

[9] Krozer V., Löffler T., Dall J., Kusk A., Eichhorn F., Olsson R.K., Buron J.D., Jepsen P.U., Zhurbenko V., Jensen T. // IEEE Trans. Microw. Theory Tech. 2010. V. 58. N 7. Pt 2. P. 2027-2039. doi 10.1109/TMTT.2010.2050246

[10] Chen H.-T., Kersting R., Cho G.C. // Appl. Phys. Lett. 2003. V. 83. N 15. P. 3009-3011. doi 10.1063/1.1616668

[11] Huber A.J., Keilmann F., Wittborn J., Aizpurua J., Hillenbrand R. // Nano Lett. 2008. V. 8. N 11. P. 3766-3770. doi $10.1021 / \mathrm{n} 1802086 \mathrm{x}$

[12] Liewald C., Mastel S., Hesler J., Huber A.J., Hillenbrand R., Keilmann F. // Optica. 2018. V. 5. N 2. P. 159. doi 10.1364/OPTICA.5.000159

[13] Lukyanchuk B.S., Paniagua-Dominguez R., Minin I.V, Minin O.V, Wang Z. // Opt. Mater. Express. 2017. V. 7. N 6. P. 1820-1847. doi 10.1364/OME.7.001820

[14] Yue L., Minin O.V., Wang Z., Monks J.N., Shalin A.S., Minin I.V. // Opt. Lett. 2018. V. 43. N 4. P. 771. doi 10.1364/OL.43.000771

[15] Minin I.V., Minin O.V., Ponomarev D.S., Glinskiy I.A. // Ann. Phys. 2018. V. 530. N 12. P. 1800359. doi 10.1002/andp.201800359

[16] Minin I.V., Minin O.V., Katyba G.M., Chernomyrdin N.V., Kurlov V.N., Zaytsev K.I., Yue L., Wang Z., Christodoulides D.N. // Appl. Phys. Lett. 2019. V. 114. N 3. P. 031105. doi 10.1063/1.5065899

[17] Yue L., Yan B., Monks J.N., Dhama R., Wang Z., Minin O.V., Minin I.V. // Ann. Phys. 2018. V. 530. N 2. P. 1700384. doi 10.1002/andp.201700384

[18] Yue L., Yan B., Monks J.N., Wang Z., Tung N.T., Lam V.D., Minin O., Minin I. // J. Phys. D. 2017. V. 50. N 17. P. 175102. doi 10.1088/1361-6463/aa61cb 
[19] Nguyen Pham H.H., Hisatake S., Minin I.V., Minin O.V., Nagatsuma T. // Appl. Phys. Lett. 2016. V. 108. N 19. P. 191102. doi 10.1063/1.4949014

[20] Nguyen Pham H.H., Hisatake S., Minin O.V., Nagatsuma T., Minin I.V. // APL Photonics. 2017. V. 2. N 5. P. 056106. doi 10.1063/1.4983114

[21] Minin I.V., Minin O.V. // Opt. Quantum Electron. 2017. V. 49. N 10. P. 326. doi 10.1007/s11082-017-1165-6

[22] Yue L., Yan B., Monks J.N., Dhama R., Wang Z., Minin O.V., Minin I.V. // J. Infrared, Millimeter, Terahertz Waves. 2018. V. 39. N 6. P. 546-552. doi 10.1007/s10762-018-0479-1

[23] Mansfield S.M., Kino G.S. // Appl. Phys. Lett. 1990. V. 57. N 24. P. 2615-2616. doi 10.1063/1.103828

[24] Terris B.D., Mamin H.J., Rugar D., Studenmund W.R., Kino G.S. // Appl. Phys. Lett. 1994. V. 65. N 4. P. 388-390. doi $10.1063 / 1.112341$

[25] Lerman G.M., Israel A., Lewis A. // Appl. Phys. Lett. 2006. V. 89. N 22. P. 1-4. doi 10.1063/1.2398888

[26] Ippolito S.B., Thorne S.A., Eraslan M.G., Goldberg B.B., Ünlü M.S., Leblebici Y. // Appl. Phys. Lett. 2004. V. 84. N 22. P. 4529-4531. doi 10.1063/1.1758308

[27] Köklü F.H., Quesnel J.I., Vamivakas A.N., Ippolito S.B., Goldberg B.B., Ünlü M.S. // Opt. Express. 2008. V. 16. N 13. P. 9501. doi 10.1364/OE.16.009501

[28] Pimenov A., Loidl A. // Appl. Phys. Lett. 2003. V. 83. N 20. P. 4122-4124. doi 10.1063/1.1627474

[29] Gompf B., Gerull M., Müller T., Dressel M. // Infrared Phys. Technol. 2006. V. 49. N 1-2. P. 128-132. doi 10.1016/j.infrared.2006.01.021

[30] Chernomyrdin N.V., Schadko A.O., Lebedev S.P., Tolstoguzov V.L., Kurlov V.N., Reshetov I.V., Spektor I.E., Skorobogatiy M., Yurchenko S.O., Zaytsev K.I. // Appl. Phys. Lett. 2017. V. 110. N 22. P. 221109. doi 10.1063/1.4984952

[31] Chernomyrdin N.V., Kucheryavenko A., Malakhov K., Schadko A., Komandin G., Lebedev S., Dolganova I., Kurlov V., Lavrukhin D., Ponomarev D., Yurchenko S., Tuchin V.V., Zaytsev K. // Proc. SPIE. 2018. V. 10716. P. 1071606 . doi $10.1117 / 12.2314464$

[32] Chernomyrdin N.V., Kucheryavenko A.S., Zaytsev K.I., Kolontaeva G.S., Katyba G.M., Karalkin P.A., Smolyanskaya O.A., Karasik V.E., Minin O.V., Parfenov V.A., Gryadunova A.A., Norkin N.E., Minin I.V. // Proceedings of SPIE / ed. Fournier C., Georges M.P., Popescu G. // Proc. SPIE. 2018. V. 10677. P. 106771Y. doi 10.1117/12.2306132

[33] Chernomyrdin N.V., Kucheryavenko A.S., Kolontaeva G.S., Katyba G.M., Dolganova I.N., Karalkin P.A., Ponomarev D.S., Kurlov V.N., Reshetov I.V., Skorobogatiy M., Tuchin V.V., Zaytsev K.I. // Appl. Phys. Lett. 2018. V. 113. N 11. P. 111102. doi 10.1063/1.5045480

[34] Guerboukha H., Nallappan K., Skorobogatiy M. // Adv. Opt. Photonics. 2018. V. 10. N 4. P. 843. doi 10.1364/AOP.10.000843

[35] Wang J., Stantchev R.I., Sun Q., Chiu T.-W., Ahuja A.T., MacPherson E.P. // Biomed. Opt. Express. 2018. V. 9. N 12. P. 6467. doi 10.1364/BOE.9.006467

[36] Ashworth P.C., Pickwell-MacPherson E., Provenzano E., Pinder S.E., Purushotham A.D., Pepper M., Wallace V.P. // Opt. Express. 2009. V. 17. N 15. P. 12444. doi 10.1364/OE.17.012444

[37] Milster T.D., Jo J.S., Hirota K. // Appl. Opt. 1999. V. 38. N 23. P. 5046. doi 10.1364/AO.38.005046
[38] Karrai K., Lorenz X., Novotny L. // Appl. Phys. Lett. 2000. V. 77. N 21. P. 3459-3461. doi 10.1063/1.1326839

[39] Komandin G.A., Chuchupal S.V., Lebedev S.P., Goncharov Y.G., Korolev A.F., Porodinkov O.E., Spektor I.E., Volkov A.A. // IEEE Trans. Terahertz Sci. Technol. 2013. V. 3. N 4. P. 440-444. doi 10.1109/TTHZ.2013.2255914

[40] Zahl H.A., Golay M.J.E. // Rev. Sci. Instrum. 1946. V. 17. N 11. P. 511-515. doi 10.1063/1.1770416

[41] Nyquist H. // Trans. Am. Inst. Electr. Eng. 1928. V. 47. N 2. P. 617-644. doi 10.1109/T-AIEE.1928.5055024

[42] Tuchin V.V. Tissue Optics: Light Scattering Methods and Instruments for Medical Diagnosis. Third Edition. Bellingham, Washington, USA: SPIE Press, 2007. 882 p. doi $10.1117 / 3.1003040$

[43] Sesek A., Zemva A., Trontelj J. // Recent Pat. Nanotechnol. 2018. V. 12. N 1. P. 34-44. doi $10.2174 / 1872210511666170704103627$

[44] Chen F.Y., Fang Y.K., Hsu C.-Y., Chen J.-R. // Ferroelectrics. 1997. V. 200. N 1. P. 257-268. doi $10.1080 / 00150199708008610$

[45] Fletcher D.A., Crozier K.B., Quate C.F., Kino G.S., Goodson K.E., Simanovskii D., Palanker D.V. // Appl. Phys. Lett. 2001. V. 78. N 23. P. 3589-3591. doi 10.1063/1.1377318

[46] Fletcher D.A., Crozier K.B., Quate C.F., Kino G.S., Goodson K.E., Simanovskii D., Palanker D.V. // Appl. Phys. Lett. 2000. V. 77. N 14. P. 2109-2111. doi 10.1063/1.1313368

[47] Mazhorova A., Markov A., Ng A., Chinnappan R., Skorobogata O., Zourob M., Skorobogatiy M. // Opt. Express. 2012. V. 20. N 5. P. 5344-5355.

[48] Fan W., Yan B., Wang Z., Wu L. // Sci. Adv. 2016. V. 2. N 8. P. 40-42. doi 10.1126/sciadv.1600901

[49] Katyba G.M., Zaytsev K.I., Chernomyrdin N.V, Shikunova I.A., Komandin G.A., Anzin V.B., Lebedev S.P., Spektor I.E., Karasik V.E., Yurchenko S.O., Reshetov I.V, Kurlov V.N., Skorobogatiy M. // Adv. Opt. Mater. 2018. V. 6. P. 1800573. doi 10.1002/adom.201800573

[50] Fan S., Ung B., Parrott E.P.J., Pickwell-MacPherson E. // Phys. Med. Biol. 2015. V. 60. N 7. P. 2703-2713. doi 10.1088/0031-9155/60/7/2703

[51] Zaytsev K.I., Chernomyrdin N.V. Gavdush A.A., Beshplav S.I.T., Malakhov K.M., Kucheryavenko A.S., Katyba G.M., Dolganova I.N., Goryaynov S.A., Karassik V.E., Spector I.E., Kurlov V.N., Yurchenko S.O., Komandin G.A., Potapov A.A., Tuchin V.V. // Proc. SPIE. 2018. V. 10716. P. 107160S. doi 10.1117/12.2316302

[52] Pickwell E., Cole B.E., Fitzgerald A.J., Wallace V.P., Pepper M. // Appl. Phys. Lett. 2004. V. 84. N 12. P. 2190 2192. doi $10.1063 / 1.1688448$

[53] Tuchin V.V., Maksimova I.L., Zimnyakov D.A., Kon I.L., Mavlutov A.H., Mishin A.A. // J. Biomed. Opt. 1997. V. 2. N 4. P. 401. doi $10.1117 / 12.281502$

[54] Ishimaru A. Electromagnetic Wave Propagation, Radiation, and Scattering: From Fundamentals to Applications. 2nd ed. Hoboken, New Jersey, USA: Wiley-IEEE Press, 2017. 968 p. 